\title{
New horizons for studying human hepatotropic infections
}

\author{
Ype P. de Jong, ${ }^{1,2}$ Charles M. Rice, ${ }^{1}$ and Alexander Ploss ${ }^{1}$
}

${ }^{1}$ Center for the Study of Hepatitis C, The Rockefeller University, New York, New York.

2Division of Gastroenterology, Mount Sinai School of Medicine, New York, New York.

\begin{abstract}
The liver serves as a target organ for several important pathogens, including hepatitis $B$ and $C$ viruses (HBV and $\mathrm{HCV}$, respectively) and the human malaria parasites, all of which represent serious global health problems. Because these pathogens are restricted to human hepatocytes, research in small animals has been compromised by the frailty of the current mouse xenotransplantation models. In this issue of the JCI, Bissig et al. demonstrate robust $\mathrm{HBV}$ and $\mathrm{HCV}$ infection in a novel xenotransplantation model in which large numbers of immunodeficient mice with liver injury were engrafted with significant quantities of human hepatocytes. This technical advance paves the way for more widespread use of human liver chimeric mice and forms the basis for creating increasingly complex humanized mouse models that could prove useful for studying immunopathogenesis and vaccine development against hepatotropic pathogens.
\end{abstract}

More than 800 million people are chronically infected with hepatitis $B$ and $C$ viruses (HBV and HCV, respectively) and malaria, causing over 1.5 million deaths annually $(1,2)$. Persistent HBV and HCV infections can lead to cirrhosis and/or hepatocellular carcinoma (HCC). Basic research, as well as the development of drugs and vaccines targeting human hepatotropic pathogens, has been handicapped by the lack of robust in vitro and in vivo platforms that mimic human liver biology and disease susceptibility. The narrow species tropism of $\mathrm{HBV}$ and $\mathrm{HCV}$ restricts preclinical studies to chimpanzees or human liver-chimeric mice. Large primate studies are often limited by financial and ethical concerns, and humanized mice are currently cumbersome and low-throughput. In culture, HCC-derived cell lines and immortalized hepatocytes have been useful for studying various aspects of HBV and HCV life cycles, but these cells differ phenotypically and functionally from human hepatocytes in vivo. Primary hepatocytes, which can be coaxed to maintain liver-specific functions by culture with fibroblasts, hold promise as a more physiologically relevant in vitro substrate for $\mathrm{HCV}$ infection (3). Addressing

Conflict of interest: C.M. Rice holds equity in Apath LLC, and AlphaVax Inc. and serves as an advisor for Genentech, GlaxoSmithKline, iTherX, Merck, Novartis Vaccines and Diagnostics, Pfizer, and Vertex.

Citation for this article: J Clin Invest. 2010; 120(3):650-653. doi:10.1172/JCI42338. fundamental questions about hepatotropic pathogen biology in vivo, however, requires a suitable small animal model to complement and guide more challenging and expensive studies, including clinical trials.

Human liver-chimeric mouse models for human hepatotropic infections

Currently, the most advanced in vivo systems for modeling human hepatotropic infectious disease are human liver-chimeric mice. A high degree of chimerism (up to $99 \%$ ) can be achieved by transplanting human hepatocytes into immunodeficient mice in which liver injury has been induced to ablate the endogenous murine hepatocytes (Figure 1). The best-characterized model to date is the immunodeficient urokinase-type plasminogen activator (uPA) mouse, in which an albumin (Alb) promoter directs high-level toxic expression of uPA (Alb-uPA mice) $(4,5)$. The hepatotoxicity creates a permissive environment for the expansion of functional, transgene-free human hepatocytes. Human liver-chimeric immunodeficient Alb-uPA mice are susceptible to hepatotropic human pathogens $(4,5)$ and have been used in studies ranging from viral evolution to preclinical testing of antiviral compounds $(6,7)$. Working with the Alb-uPA model, however, poses serious challenges (Table 1). The selective pressure needed to achieve and maintain a high level of human chimerism can only be achieved in homozygous Alb-uPA immunodeficient recipients, which suffer from infertility. Therefore large, costly, hemizygous breeder cohorts, or rescue of breeder pairs by transplantation with normal hepatocytes, is required to propagate these mice (8). The severe liver injury of homozygous AlbuPA immunodeficient pups necessitates engraftment surgery in the first weeks of life - a procedure that is complicated by the susceptibility of these animals to fatal hemorrhaging (9). Because of these factors, along with the need for high-quality adult human hepatocytes, only modest numbers of experimental mice can be generated, and at great expense.

To overcome some of these challenges, alternative liver injury models have been developed, including a less toxic Alb-uPA line (10), mice characterized by inducible uPA transgene expression (11), or mice in which the major urinary protein (MUP) promoter is used to delay uPA expression (12). Recently, two groups have reported successful engraftment of human hepatocytes into fumaryl acetoacetate hydrolase-deficient (FAH-deficient) mice bred with mice of a Rag2-/-Il2rgnull immunodeficient background (FRG mice) $(13,14)$ (Table 1). FAH is the last enzyme in the tyrosine breakdown pathway (Figure 2), and its deficiency leads to lethal type 1 hypertyrosinemia in humans and liver failure in mice. Treatment with 2-(2-nitro-4trifluoromethylbenzyol)-cyclohexane-1,3dione (NTBC) prevents the accumulation of toxic metabolites and hepatotoxicity (15), allowing liver injury to be induced at will by withdrawal of the drug.

Using mice of the FRG background, Bissig et al. report consistently high, on average $42 \%$, human liver chimerism after transplantation of human adult hepatocytes (Table 1) (16). The trick to achieving this high chimerism seems to be the use of 3-5 times more human adult hepatocytes than in previous studies $(13,14)$. The impressively large number of successfully transplanted animals revealed a clear correlation between the level 


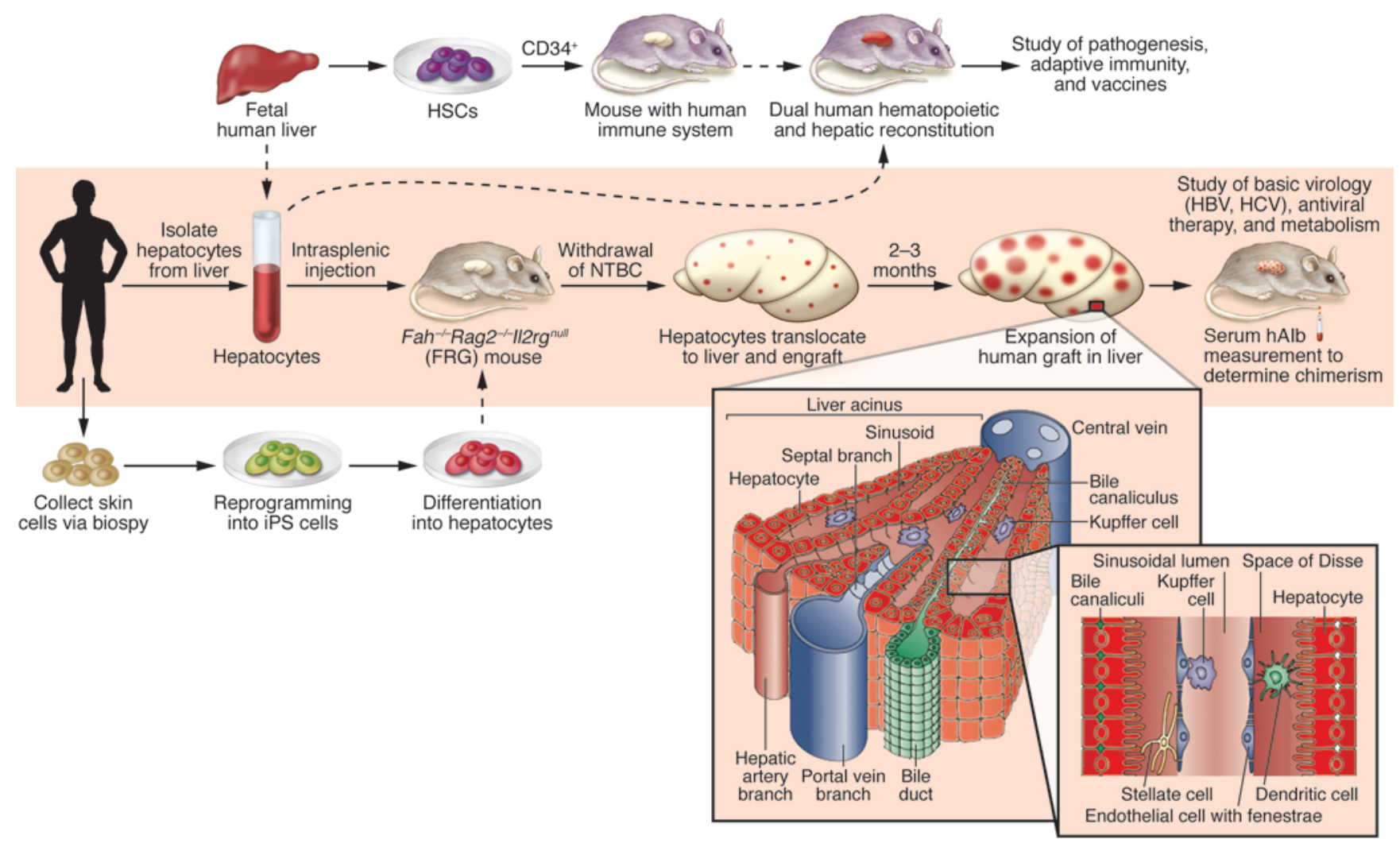

Figure 1

Creation and future improvements of human liver-chimeric FRG mice. In the model described in the current issue by Bissig et al. (16), enclosed in the shaded rectangle, hepatocytes are isolated from adult human liver tissue and injected into FRG mice that are cycled off the protective drug NTBC. Human hepatocyte engraftment levels are then monitored by serial human albumin (hAlb) measurements in the serum of transplanted mice. Over 2-3 months, the human hepatocytes expand and can repopulate up to 97\% of the FRG mouse liver, with the remainder of liver-resident cells likely of murine origin. This robust system can be used to study HBV and HCV infections in vivo and can serve as a scaffold for more complex humanized mouse models. The engrafted FRG mice reported by Bissig et al. could potentially be combined with mice bred to posses a human immune system, which are generated by transplantation of human CD34+ stem cells (HSCs) (23). Use of donor hepatocytes and HSCs derived from fetal liver may allow FRG mice animals to be repopulated with the immune system and liver tissue of an individual human donor, facilitating studies of immunopathogenesis and vaccine testing. Another use for the FRG model will be to study hepatocyte differentiation from human induced pluripotent stem (iPS) cells $(24,25)$ derived from dermal fibroblasts or from embryonic stem cells $(26)$. Steps depicted by solid lines are currently feasible; the dashed lines refer to steps that are under investigation but have not yet proven workable. Figure modified with permission from Hepatology (27), Nature Reviews Immunology (28), and Cell Host \& Microbe (22) and based on concepts discussed in ref. 29.

of chimerism and human serum albumin - providing a convenient, minimally invasive test to monitor engraftment (16). The authors then tested whether the engrafted animals were susceptible to $\mathrm{HBV}$ and $\mathrm{HCV}$ infection. Similar to previous studies with immunodeficient Alb-uPA mice $(5,17)$, FRG mice were shown to be susceptible to HBV infection, irrespective of the level of human chimerism. Hallmarks of viral replication, including the presence of serum HBV DNA, covalently closed circular HBV DNA, and $\mathrm{HBV}$ core antigen in liver tissue, were readily observed. In contrast, successful HCV infection could only be achieved if more than $10 \%$ of hepatocytes were of human origin, again in line with previous observations in immunodeficient Alb-uPA mice (4, 5). Inter- estingly, above this $10 \%$ threshold of chimerism, no clear correlation between hepatocyte engraftment levels and serum viremia was observed. One can only speculate as to why HCV requires substantially higher human chimerism - the short in vivo halflife of the virus may require rapid uptake by new permissive target cells, or successful propagation may necessitate densities of human hepatocytes sufficient for cell-to-cell spread. Importantly, HCV infection could be initiated using cell culture-grown or patient-derived virus, with the highest viremia level achieved using a patient-derived isolate. Furthermore, HCV antigens could be detected in the livers of infected animals, traditionally a highly technically difficult achievement (18).

\section{Potential of human liver-chimeric mice in preclinical drug development} The susceptibility of humanized FRG mice to hepatotropic infections holds promise for preclinical drug efficacy testing. The authors demonstrate a modest decrease in HBV viremia after short-term treatment with the reverse transcriptase inhibitor adefovir dipivoxil, similar to the slow kinetics of viral decline seen in humans treated with this drug (16). For $\mathrm{HCV}$, the current treatment is pegylated IFN- $\alpha$ (peg-IFN) and ribavirin, a combination therapy that is often poorly tolerated and unsuccessful. Several drug candidates targeting viral and host proteins are in preclinical or clinical development, and a major question is whether the virus 
Table 1

Comparison between FRG mice and immunodeficient Alb-uPA human liver-chimeric mice

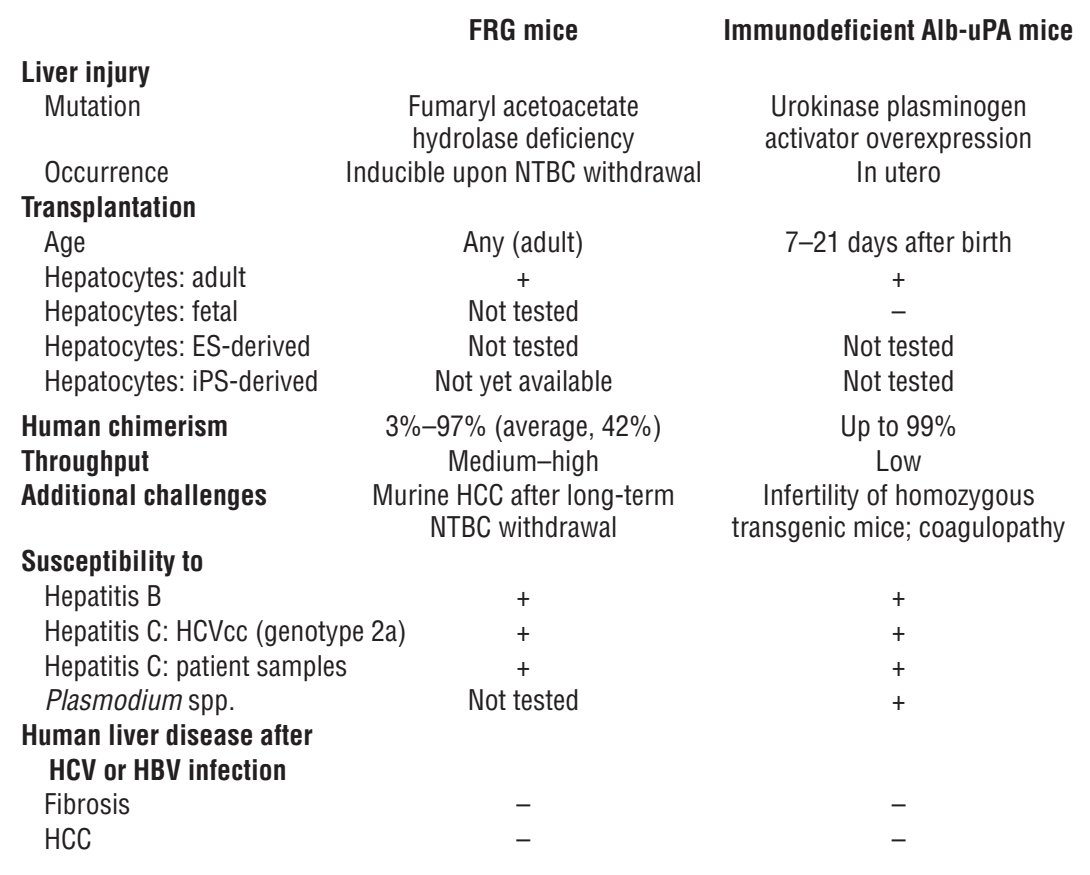

iPS, induced pluripotent stem; HCVcc, cell culture-derived HCV.

can be eliminated using solely a cocktail of specific antivirals. Identification of the most effective drug combinations, and the ability to monitor resistance, is an area where model animals could have great utility. Bissig et al. show that a combination of peg-IFN and Debio 025 - an HCV inhibitor targeting the host factor cyclophilin $\mathrm{A}-$ is as effective as peg-IFN plus ribavirin over a short-term treatment period. Overall, the results of the Bissig et al. study recapitulate many aspects of HBV and HCV infection in humans, illustrating the promise of this new model for basic studies of human hepatitis viruses and preclinical drug efficacy assessments.

\section{The next steps}

The development of more robust human liver-chimeric mice is an important step forward, but further refinements are needed. Despite prolonged high viremia, none of the commonly observed sequelae associated with $\mathrm{HBV}$ or $\mathrm{HCV}$ infections in humans, namely fibrosis or

\section{Figure 2}

Tyrosine metabolic pathway affected by $\mathrm{FAH}$ deficiency, and the step in this pathway that is inhibited by NTBC.
HCC, were observed in the current study (16). (Patho)physiological processes may require crosstalk between hepatocytes and other liver-resident cells, and, while human hepatocytes are abundant in highly engrafted chimeric mice, nonparenchymal cells are of murine-recipient origin. Kupffer cells and liver sinusoidal endothelial cells, for example, appear to be critical to the ability of Plasmodium sporozoites (the transmission form of

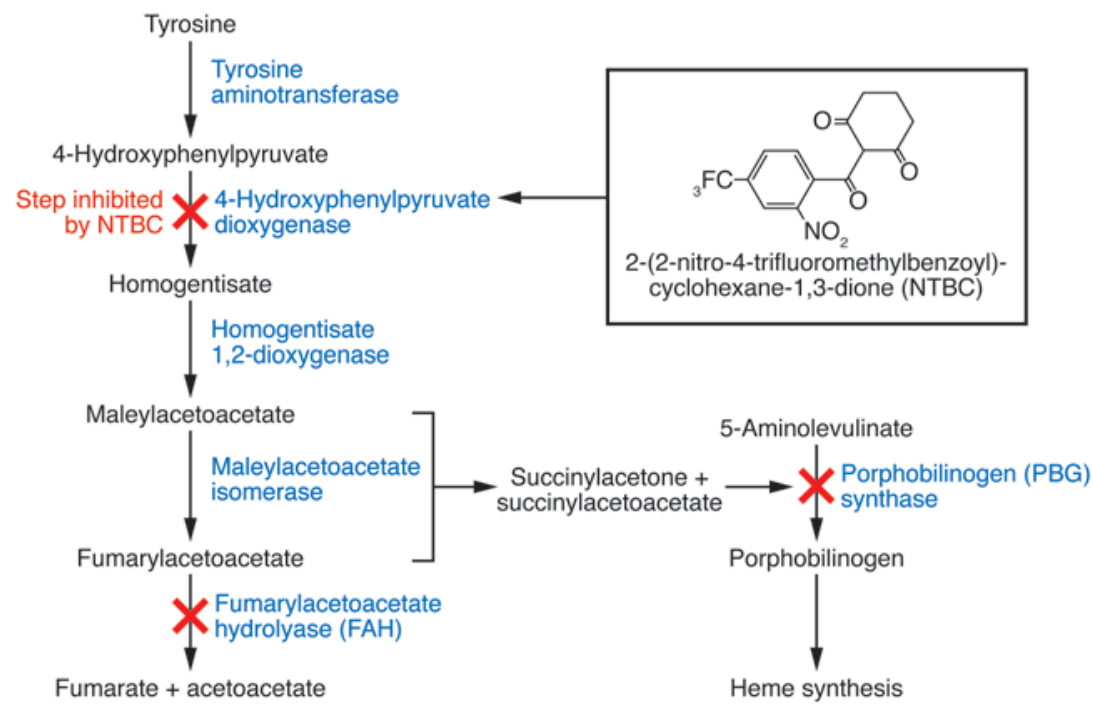

the malaria parasite) to infect the liver, as permissive hepatocytes are not directly accessible to the sporozoites (19). While infection of human liver-chimeric mice with virulent and live-attenuated Plasmodium species has been reported $(20,21)$, infection frequencies were low and may in the future possibly be improved by addition of other human non-parenchymal liver cell subsets.

Another obvious caveat of the current human liver-chimeric models is their immunodeficient background. Chronic inflammation is thought to be a major contributor to fibrosis during persistent HBV and HCV infection. Thus, dual engraftment of these animals, resulting in a human liver and a human immune system, is not only critical to monitor immune responses to infection, but also to more faithfully mimic disease pathogenesis (22). Furthermore, since viral hepatitis has become the leading morbidity in HIV-infected individuals in the Western world, modeling such coinfections in dually reconstituted mice would be another clinically important application (Figure 1).

In summary, human liver-chimeric mice can serve as valuable tools in preclinical drug efficacy, toxicity, and pharmacokinetic applications but also facilitate the study of human hepatotropic pathogens. After more than a decade of working with frail immunodeficient Alb-uPA mice, the FRG model reported by Bissig and colleagues lends itself to become the cornerstone for a wide variety of investigations and will likely provide the platform for further improvements (16). 


\section{Acknowledgments}

The authors thank Catherine Murray (The Rockefeller University) for editing the manuscript. This work was supported by grants from the Gates Foundation through the Grand Challenges in Global Health initiative (to all authors) and funded in part by the Greenberg Medical Research Institute, the Ellison Medical Foundation, the Starr Foundation, the Ronald A. Shellow Memorial Fund, the Richard Salomon Family Foundation (to C.M. Rice), and the NIH through the NIH Roadmap for Medical Research, grant 1 R01 DK085713-01 (to C.M. Rice and A. Ploss). C.M. Rice is an Ellison Medical Foundation Senior Scholar in Global Infectious Diseases.

Address correspondence to: Alexander Ploss or Charles M. Rice, Center for the Study of Hepatitis C, The Rockefeller University, 1230 York Avenue, Box 64, New York, NY 10065. Phone: 212.327.7066; Fax: 212.327.7048; E-mail: aploss@rockefeller.edu (A. Ploss). Phone: 212.327.7046; Fax: 212.327.7048; E-mail: ricec@rockefeller.edu (C.M. Rice).

1. Alter MJ. Epidemiology and prevention of hepatitis B. Semin Liver Dis. 2003;23(1):39-46.

2. Shepard CW, Finelli L, Alter MJ. Global epidemiology of hepatitis C virus infection. Lancet Infect Dis. 2005;5(9):558-567.

3. Ploss A, et al. Persistent hepatitis $C$ virus infection in microscale primary human hepatocyte cultures. Proc Natl Acad Sci U S A. In press.

4. Mercer DF, et al. Hepatitis C virus replication in mice with chimeric human livers. Nat Med. 2001;7(8):927-933.

5. Meuleman P, et al. Morphological and biochemical characterization of a human liver in a UPA-SCID mouse chimera. Hepatology. 2005;41(4):847-856.

6. Kaul A, Woerz I, Meuleman P, Leroux-Roels G, Bartenschlager R. Cell culture adaptation of hepatitis $\mathrm{C}$ virus and in vivo viability of an adapted variant. J Virol. 2007;81(23):13168-13179.

7. Meuleman P, Leroux-Roels G. The human liveruPA-SCID mouse: a model for the evaluation of antiviral compounds against $\mathrm{HBV}$ and HCV. Antiviral Res. 2008;80(3):231-238.

8. Brezillon NM, et al. Rescue of fertility in homozygous mice for the urokinase plasminogen activator transgene by the transplantation of mouse hepatocytes. Cell Transplant. 2008;17(7):803-812.

9. Heckel JL, Sandgren EP, Degen JL, Palmiter RD, Brinster RL. Neonatal bleeding in transgenic mice expressing urokinase-type plasminogen activator. Cell. 1990;62(3):447-456.

10. Suemizu H, et al. Establishment of a humanized model of liver using NOD/Shi-scid IL2Rgnull mice. Biochem Biophys Res Commun. 2008;377(1):248-252.

11. Song $X$, et al. A mouse model of inducible liver injury caused by tet-on regulated urokinase for studies of hepatocyte transplantation. Am J Pathol. 2009;175(5):1975-1983.

12. Weglarz TC, Degen JL, Sandgren EP. Hepatocyte transplantation into diseased mouse liver. Kinetics of parenchymal repopulation and identification of the proliferative capacity of tetraploid and octaploid hepatocytes. Am J Pathol. 2000;157(6):1963-1974

13. Azuma H, et al. Robust expansion of human hepatocytes in Fah-/-/Rag2-/-/Il2rg-/- mice. Nat Biotechnol. 2007;25(8):903-910.

14. Bissig KD, Le TT, Woods NB, Verma IM. Repopulation of adult and neonatal mice with human hepatocytes: a chimeric animal model. Proc Natl Acad Sci U S A. 2007;104(51):20507-20511.

15. Grompe $M$, et al. Pharmacological correction of neonatal lethal hepatic dysfunction in a murine model of hereditary tyrosinaemia type I. Nat Genet. 1995;10(4):453-460.
16. Bissig K-D, et al. Human liver chimeric mice provide a model for hepatitis $\mathrm{B}$ and $\mathrm{C}$ virus infection and treatment. J Clin Invest. 2010;120(3):924-930.

17. Dandri $M$, et al. Repopulation of mouse liver with human hepatocytes and in vivo infection with hepatitis B virus. Hepatology. 2001;33(4):981-988.

18. Liang $\mathrm{Y}$, et al. Visualizing hepatitis $\mathrm{C}$ virus infections in human liver by two-photon microscopy. Gastroenterology. 2009;137(4):1448-1458.

19. Frevert U, Usynin I, Baer K, Klotz C. Plasmodium sporozoite passage across the sinusoidal cell layer. Subcell Biochem. 2008;47:182-197.

20. VanBuskirk KM, et al. Preerythrocytic, liveattenuated Plasmodium falciparum vaccine candidates by design. Proc Natl Acad Sci U S A. 2009;106(31):13004-13009.

21. Morosan S, et al. Liver-stage development of Plasmodium falciparum, in a humanized mouse model. J Infect Dis. 2006;193(7):996-1004.

22. Legrand $N$, et al. Humanized mice for modeling human infectious disease: challenges, progress, and outlook. Cell Host Microbe. 2009;6(1):5-9.

23. Shultz LD, Ishikawa F, Greiner DL. Humanized mice in translational biomedical research. Nat Rev Immunol. 2007;7(2):118-130.

24. Sullivan GJ, et al. Generation of functional human hepatic endoderm from human induced pluripotent stem cells. Hepatology. 2010;51(1):329-335.

25. Si-Tayeb K, et al. Highly efficient generation of human hepatocyte-like cells from induced pluripotent stem cells. Hepatology. 2010;51(1):297-305.

26. Snykers S, De Kock J, Rogiers V, Vanhaecke T. In vitro differentiation of embryonic and adult stem cells into hepatocytes: state of the art. Stem Cells. 2009;27(3):577-605.

27. Kneteman NM, Mercer DF. Mice with chimeric livers: who says supermodels have to be tall? Hepatology. 2005;41(4):703-706.

28. Adams DH, Eksteen B. Aberrant homing of mucosal $\mathrm{T}$ cells and extra-intestinal manifestations of inflammatory bowel disease. Nat Rev Immunol. 2006;6(3):244-251.

29. Ploss A, Rice CM. Towards a small animal model for hepatitis C. EMBO Rep. 2009;10(11):1220-1227.

\title{
Bidirectional homing of Tregs between the skin and lymph nodes
}

\author{
Hironori Matsushima and Akira Takashima
}

Department of Medical Microbiology and Immunology, University of Toledo College of Medicine, Ohio.

\begin{abstract}
Although several homing receptors are known to be differentially expressed by Tregs in lymphoid tissues compared with those found in peripheral tissues, it remains unclear whether these cells traffic between the two locations. In this issue of the JCI, Tomura et al. report steadystate Treg migration from the skin to draining LNs in mice. Furthermore, they report that not only does skin inflammation exacerbate LN-directed Treg homing, it also triggers reverse circulation of Tregs from LNs to skin, whereby these cells contribute to regulation of the immune response. These results now form a new framework for our understanding of Treg homing.
\end{abstract}

Conflict of interest: The authors have declared that no conflict of interest exists.

Citation for this article: J Clin Invest. 2010; 120(3):653-656. doi:10.1172/JCI42280.
Memory T cells are disseminated to lymphoid and nonlymphoid tissues throughout the body, and their migration to respective tissues is tightly regulated by adhesion mol- ecules and chemokine receptors (Table 1). For example, memory $\mathrm{T}$ cells that infiltrate the skin express a unique adhesion molecule, known as cutaneous lymphocyte-associated antigen (CLA), which is produced from P-selectin glycoprotein ligand-1 through posttranscriptional carbohydrate modification by fucosyltransferase VII. Skin-homing memory T cells also express specific chemokine receptors: CCR4, CCR6, and CCR10. In contrast, memory $T$ cells that preferentially circulate through lymphoid tissues express CD62 ligand (CD62L; also known as L-selectin) and CCR7 (1). 